\title{
Governance in der Staatsschuldenkrise
}

\author{
Die Eurokrise hat tiefgreifende Veränderungen im Gefüge von Institutionen und Kompetenzen \\ in der Europäischen Union angestoßen, dafür stehen der „Fiskalpakt“ und die „excessive \\ imbalance procedure“. Ein effizientes Governance-System hat sich aber bisher nicht \\ herausgebildet. Die einzelnen Politikbereiche sind zudem innerhalb der EU unterschiedlich \\ stark integrierbar. Zwischen Bereichen, in denen Zentralisierungstendenzen wünschenswert \\ sind und solchen, in denen das Subsidiaritätsprinzip angemessen ist, muss klar unterschieden \\ werden. Bei der Krisenbewältigung müssen demokratische Prinzipien dringend stärker
} beachtet werden.

\section{Illegitim und rechtswidrig: Das neue makroökonomische Regime im Euroraum}

Die Eurokrise hat tiefgreifende Veränderungen im Gefüge von Institutionen und Kompetenzen der Europäischen Union angestoßen. Als Reaktion auf die Krise erfolgte nicht nur eine Verschärfung des Stabilitätspakts, der künftig automatisierte Sanktionen vorsieht und der auf Grundlage des zwischenstaatlichen „Fiskalpakts“ um nationale Schuldenbremsen nach deutschem Vorbild ergänzt werden soll. Installiert wurde zudem auch ein „Verfahren zur Vermeidung und Korrektur makroökonomischer Ungleichgewichte" (Excessive Imbalance Procedure - EIP). Künftig wird die Europäische Kommission jährlich länderbezogene Wirtschaftsdaten auf Anzeichen für sich entwickelnde Fehlentwicklungen prüfen und bei Erreichen eines gewissen (Über-)Maßes ein Korrekturverfahren gegen den betroffenen Mitgliedstaat eröffnen. Der Ablauf ist dem Verfahren bei übermäßigen Defiziten nach Art. 126 AEUV nachgebildet. Hat der betroffene Mitgliedstaat die Korrekturvorgaben mangelhaft umgesetzt und gehört er der Eurozone an, können die Unionsorgane Sanktionen in Gestalt von Geldbußen verhängen.

Drei Punkte sind hierbei von besonderer Brisanz.

1. Die Korrekturvorgaben können ohne Rücksicht auf die Grenzen der regulativen Kompetenzen der EU erfolgen und im Prinzip alle Politikbereiche betreffen, inklusive der Lohn-, Sozial- und Arbeitsmarktpolitik sowie der allgemeinen Ausrichtung der Budgetpolitik - Politikbereiche also, deren Gestaltung nach der europäischen Kompetenzordnung dem demokratischen Prozess innerhalb der Mitgliedstaaten vorbehalten ist.
2. Das Verfahren setzt eine bemerkenswerte Machtverlagerung vom (intergouvernementalen) Rat zur (supranationalen) Kommission ins Werk. Zwar fällt die Beschlussfassung über einzuleitende Gegenmaßnahmen und Sanktionen weiterhin formell dem Rat zu. Tatsächlich aber ist seine Gestaltungsmacht an wesentlichen Stellen des Verfahrens auf ein Vetorecht gegenüber den Beschlussvorlagen der Kommission verkürzt.

3. Mit der EIP operiert die Union in einem Steuerungsmodus, der mit dem Ziel der schrittweisen Harmonisierung durch europäische Gesetzgebung nichts mehr zu tun hat. Die „Europäische Wirtschaftsregierung“ im Rahmen der EIP beruht vielmehr auf partikularen, also an einzelne Mitgliedstaaten gerichteten Weisungen zur Umsetzung von Vorgaben der Unionsorgane.

\section{Makroökonomische Spannungen im Euroraum}

Warum haben sich die Mitgliedstaaten zur Ingangsetzung dieses bemerkenswerten Verfahrens entschlossen? Über die Ursachen der erheblichen makroökonomischen Spannungen, die sich seit der Einführung der gemeinsamen Währung im Euroraum gebildet haben, ist viel gesagt und geschrieben worden. Wir wollen es daher bei knappen Hinweisen belassen: Im Kern besteht die Schwierigkeit in einer weit geöffneten Schere 
aus markant erhöhten Anforderungen an die makroökonomische Performance der Mitgliedstaaten einerseits bei gleichzeitigem Wegfall mehrerer zur Zielerreichung eigentlich notwendiger Steuerungsinstrumente andererseits. Die europäische Zinspolitik setzt für die betroffenen Mitgliedstaaten dysfunktionale Impulse, weil sich der einheitliche nominale Zinssatz der EZB angesichts divergenter Inflationsraten in unterschiedliche Realzinsen übersetzt. Diese unterschiedlichen Impulse treiben die europäischen Konjunkturen auseinander, statt eine Konvergenz der Konjunkturverläufe zu induzieren. Vor diesem Hintergrund wären die Mitgliedstaaten eigentlich gefordert, mit aktiver makroökonomischer Politik gegenzusteuern. Als Instrumente der makroökonomischen Steuerung stehen Volkswirtschaften normalerweise Wechselkursanpassungen, die Zinspolitik, die Fiskalpolitik und die Lohnpolitik zur Verfügung. Unter den Bedingungen der einheitlichen Währung und des verschärften Stabilitätspakts sind allerdings drei dieser vier Instrumente nicht mehr verfügbar. Allein die Lohnpolitik wäre auf der Ebene der Mitgliedstaaten im Prinzip noch zur makroökonomischen Gegensteuerung einzusetzen. Kurz, Anpassungsleistungen müssten in erster Linie durch die „richtige“ Lohnpolitik erbracht werden.

Soweit die Theorie. In der Praxis finden sich im Euroraum 17 ganz unterschiedliche Regime der Lohnaushandlung. In manchen von ihnen erfolgt die Lohnfindung durch Aushandlungen starker Verbände, in anderen hingegen dezentral und unkoordiniert. So ist die Fähigkeit zum strategischen Einsatz der Lohnpolitik, um mittelfristige Ziele zu erreichen, über den Euroraum höchst unterschiedlich verteilt. Deutschland, das nach der Euro-Einführung mit zu hohen Realzinsen konfrontiert war, reagierte auf diese Problemlage mit strategischer Lohnzurückhaltung. So gelang es, die Wirtschaft über den gezielten Aufbau von Exportüberschüssen anzukurbeln. Ländern mit unkoordinierter und deshalb kaum steuerbarer Lohnfindung steht dieser Weg der Problembewältigung nicht zur Verfügung. In der Folge haben sich die Lohnstückkosten und deshalb auch die preislichen Wettbewerbspositionen unter den Teilnehmern der Europäischen Währungsunion sehr unterschiedlich entwickelt und damit zu den erheblichen makroökonomischen Spannungen beigetragen, die wir derzeit beobachten.

Auch die Lohnpolitik lässt sich also zum Ausgleich der Ungleichgewichte im Euroraum nur bedingt einsetzen. Sie ist vielmehr ursächlich für weitere Spannungen. Vor diesem Hintergrund erscheint verständlich, dass die Mitgliedstaaten den gewagten Weg institutioneller Reformen und einer erheblichen Einschränkung ihrer Autonomie gehen, um makroökonomische Fehlentwicklungen durch zentrale, von Mitgliedstaat zu Mitgliedstaat variie- rende Vorgaben eindämmen zu können. Und ebenso erscheint es nahezu zwingend, dass das neue Instrumentarium erstmals auch den sensiblen Bereich der Lohnpolitik umfasst, sowohl auf der Ebene der zu evaluierenden Indikatoren als auch als potenzielles Ziel sanktionsbewehrter Vorgaben.

Warum sich die betroffenen Mitgliedstaaten wehren werden

Allerdings erwarten wir nicht, dass das neue makroökonomische Regime reibungslos funktionieren wird. Vielmehr befürchten wir, dass die EIP erhebliche Zwietracht unter den Teilnehmern der Europäischen Währungsunion säen wird und dass sich die künftig betroffenen Mitgliedstaaten mit den ihnen zur Verfügung stehenden, d.h. auch juristischen Mitteln gegen die über sie verhängten Sanktionen wehren werden.

Das erste von zwei Problemen liegt in der zu erwartenden asymmetrischen Behandlung der Mitgliedstaaten. Grundsätzlich sind Wirtschaftspolitiken, die im Ergebnis zu chronischen Leistungsbilanzüberschüssen führen, für die makroökonomischen Ungleichgewichte im Euroraum ebenso verantwortlich wie solche, die zu Defiziten führen. Folglich hatte sich die Aufmerksamkeit bei der Aushandlung der EIP in starkem Maße auf die Frage konzentriert, ob sich das Verfahren gleichermaßen auf die Eindämmung sowohl von Defiziten als auch von Überschüssen richten würde. Hoffnungen, die auf eine solche Symmetrie gerichtet waren, wurden aber alsbald zunichte gemacht.

Zwar beinhaltet das für die Überwachung durch die Kommission maßgebliche Scoreboard, das die Schwellenwerte makroökonomischer Indikatoren enthält, oberhalb derer wirtschaftliche Entwicklungen als Ungleichgewichte klassifiziert werden sollen, in der Tat sowohl einen Schwellenwert für übermäßige Leistungsbilanzdefizite als auch für Überschüsse. Der Schwellenwert für Überschüsse wurde - maßgeblich auf deutschen Druck - mit 6\% aber höher gelegt als die 4\%-Hürde, oberhalb derer Defizite als übermäßig angesehen werden. Auf gleicher Linie stellte Währungskommissar Olli Rehn bereits am 4. November 2011 in einem an die Finanzminister gerichteten Schreiben klar, dass der Fokus der EIP auf den Defizitländern liegen würde. Im Februar 2012 veröffentlichte die Kommission ihren ersten Bericht über makroökonomische Ungleichgewichte. Zwölf Länder sind betroffen, in allen Fällen handelt es sich um Defizitländer. Deutschland wird trotz langjähriger exorbitanter Exportüberschüsse nicht genannt. Dies begründete Kommissar Rehm mit den Worten, der Euroraum brau- 
che Volkswirtschaften, die wettbewerbsfähige Produkte anbieten können. ${ }^{1}$

Das Problem ist nun, dass die EIP vor diesem Hintergrund keine wirtschaftspolitische Neutralität für sich beanspruchen kann. Vielmehr drückt sie spezifische Länder - solche mit Leistungsbilanzdefiziten - in Richtung spezifischer Problembewältigungsstrategien, namentlich Lohnmoderation, Arbeitsmarktflexibilisierung und soziale Einschnitte. Wir wollen uns an dieser Stelle einer Einschätzung darüber enthalten, inwieweit diese Instrumente zum nachhaltigen Abbau von Außenhandelsdefiziten tatsächlich geeignet sind. Die Befunde sind bekanntlich widersprüchlich und Gegenstand kontroverser Debatten zwischen den widerstreitenden wirtschaftswissenschaftlichen Schulen. Aus Sicht der betroffenen Mitgliedstaaten aber erscheint offensichtlich, dass derart einseitige Vorgaben und die mit innen künftig sogar verknüpften Sanktionen als Gängelung empfunden werden dürften - als illegitime Fremdbestimmung zur Bewältigung einer Problemlage, die zudem nur teilweise selbst verschuldet ist.

Das zweite Problem resultiert aus der in vielen Mitgliedstaaten fehlenden und auch durch die EIP naturgemäß nicht erhöhten politischen Steuerbarkeit der Lohnentwicklung. Das Scoreboard legt fest, dass die Kommission Verfahren gegen Mitgliedstaaten eröffnen kann, wenn deren Lohnstückkosten in einem Drei-Jahres-Zeitraum um mehr als $9 \%$ gestiegen sind. Ergreift der betroffene Mitgliedstaat keine durchgreifenden Maßnahmen, können - wenn der Rat nicht mit qualifizierter Mehrheit widerspricht - Sanktionen verhängt werden. Für die betroffenen Regierungen ist die Lohnentwicklung aber nicht unmittelbar beeinflussbar, und auch die mittelbare Beeinflussung dieser Zielgröße über die freiwillige Einbindung der Gewerkschaften in sogenannte „Soziale Pakte“ (man spricht hier auch vom „liberalen Korporatismus") gelingt nur unter günstigen, historisch gewachsenen Bedingungen. Und politischen Eingriffen etwa in die Verbindlichkeit oder die Reichweite von Tarifverträgen können Garantien des nationalen Verfassungsrechts, aber auch des internationalen Menschenrechts (Europäische Menschenrechtskonvention, ILO-Konventionen) entgegenstehen. Kurz, die EIP sieht Sanktionen für Fehlentwicklungen bei Indikatoren vor, die in vielen Fällen allenfalls schwach steuerbar sind und deren Freiheit vor politischen Eingriffen in gewissem Umfang sogar rechtlich garantiert ist. Wer über etwas politisches Gespür verfügt, sollte nicht überrascht sein, wenn sich im Ernstfall herausstellt, dass dies von den Betroffenen nicht als legitim empfunden wird.

1 Vgl. Frankfurter Allgemeine Zeitung vom 15.2.2012, S. 11.
Werden also am Ende der bereits angelaufenen EIP tatsächlich Sanktionen verhängt, dann erwarten wir, dass sich die betroffenen Regierungen - die nicht mehr dieselben sein müssen wie jene, die die EIP in Kraft gesetzt haben - zur Wehr setzen werden. Diesen Regierungen steht der Weg der Nichtigkeitsklage vor dem Europäischen Gerichtshof (EuGH) offen. Ein wichtiger Angriffspunkt wird dabei die Frage sein, ob die EIP im Einklang mit den Kompetenzen der EU steht.

\section{Die EIP als Überschreitung der Unionskompetenzen}

Den rechtlichen Rahmen des Verfahrens der EIP bilden zwei förmlich voneinander unabhängige Verordnungen des Europäischen Parlaments und des Rates. Die erste ist die Verordnung (EU) Nr. 1176/2011, „über die Vermeidung und Korrektur makroökonomischer Ungleichgewichte", die auf sämtliche Mitgliedstaaten Anwendung findet, die zweite ist die Verordnung (EU) Nr. 1174/2011 „über Durchsetzungsmaßnahmen zur Korrektur übermäBiger Ungleichgewichte im Eurowährungsgebiet", welche nur die Mitglieder der Eurozone betrifft.

Die erste Verordnung regelt das Verfahren der makroökonomischen Überwachung von der generellen Überwachung der makroökonomische Situation in den Mitgliedstaaten seitens der Kommission über die Eröffnung von Verfahren gegen einzelne Staaten wegen übermäBiger Ungleichgewichte bis zu dem Moment einer Feststellung, dass ein Mitgliedstaat den ihm von Kommission und Rat vorgegebenen Korrekturmaßnahmenplan nicht eingehalten hat. Die zweite Verordnung liefert die rechtliche Grundlage dafür, dass die Mitglieder der Eurozone anschließend mit Geldbußen belegt werden können. Die Frage, ob diese zweite Verordnung (EU) Nr. 1174/2011 (im Folgenden: DurchsetzungsVO) im Kompetenzbereich der Union liegt, wird also im Zentrum der Aufmerksamkeit stehen, wenn sich einzelne Mitgliedstaaten wie von uns erwartet vor dem EuGH gegen innen gegenüber verhängte Sanktionen wehren.

Der Erlass der DurchsetzungsVO wurde auf die Vorschrift des Art. 121 Abs. 6 AEUV gestützt. Diese Vorschrift erlaubt es Rat und Europäischem Parlament, die Einzelheiten des Verfahrens multilateraler Überwachung festzulegen, das wiederum ein zentraler Bestandteil der wirtschaftspolitischen Koordinierung auf Unionsebene ist. Die Eckpunkte des Verfahrens multilateraler Überwachung finden sich in Art. 121 Abs. 3 und 4 AEUV, und diese regeln einen Prozess von Berichten, Bewertungen und Empfehlungen. Sanktionen gegenüber den Mitgliedstaaten, die den Empfehlungen nicht Folge leisten, sind an dieser Stelle nicht vorgesehen. Es ist klar und aner- 
kannt, dass solche Sanktionen darum auch nicht durch eine Verordnung auf Basis von Art. 121 Abs. 6 AEUV eingeführt werden können.

Aus diesem Grund wurde in der DurchsetzungsVO bei der obligatorischen Angabe der Kompetenzgrundlage zusätzlich zu Art. 121 Abs. 6 AEUV auch noch die Vorschrift des mit dem Vertrag von Lissabon in den Vertrag aufgenommenen Art. 136 AEUV angesprochen. Art. 136 Abs. 1 b) AEUV ermächtigt den Rat, für die Mitglieder der Eurozone besondere Grundzüge der Wirtschaftspolitik zu erarbeiten und deren Einhaltung zu überwachen. Entscheidend ist daher die Frage, ob Art. 136 AEUV den Rat im Rahmen der dort angesprochenen Überwachung über unverbindliche Empfehlungen hinaus auch zu Sanktionen ermächtigt. Wäre diese Frage zu bejahen, hätten Rat und EP wohl tatsächlich auf Basis von Art. 121 Abs. 6 AEUV die Einzelheiten der Überwachung in der DurchsetzungsVO festlegen dürfen.

Doch die Frage ist eindeutig zu verneinen. Art. 136 AEUV ermächtigt den Rat nicht zu Sanktionen, sondern nur zu Maßnahmen von der Art, die sich bereits in den Eckpunkten des Verfahrens multilateraler Überwachung nach Art. 121 Abs. 3 und 4 AEUV finden. Eine sorgfältige Auslegung der zunächst etwas unverständlich anmutenden Vorschrift des Art. 136 AEUV lässt am Ende keinen Zweifel: Zum einen regelt Art. 136 AEUV kein Verfahren zur Annahme der Sanktionsbeschlüsse durch den Rat, wie es an sich erforderlich wäre. Auch Art. 121 AEUV, auf den Art. 136 AEUV generell für die Annahmeverfahren verweist, enthält kein Verfahren für Sanktionen, sondern naturgemäß nur Verfahren für die dort vorgesehenen unverbindlichen Ratsmaßnahmen von Bewertung und Empfehlung. Zum anderen würde eine entgegengesetzte Auslegung letztlich darauf hinauslaufen, dass der Union bereits mit der Aufnahme von Art. 136 AEUV in den Vertrag eine umfassende Kompetenz zur Steuerung der mitgliedstaatlichen Wirtschaftspolitik im weitesten Sinne, also unter Einschluss von Steuer-, Arbeits- und Sozialpolitik, und nicht nur in Gestalt von Sanktionen, sondern auch in Gestalt europäischer Gesetzgebung zugesprochen worden wäre. Die Union verfügte also seit Inkrafttreten des Vertrages von Lissabon im Jahr 2009 über die rechtlichen Grundlagen einer echten Wirtschaftsregierung, nur dass das bis heute unbemerkt geblieben wäre. Das erscheint jenseits des Vertretbaren.

Ob freilich der EuGH in einer mit Blick auf den Bestand des Euro womöglich als notstandsähnlich wahrgenommenen Situation die an sich offensichtliche Kompetenzüberschreitung der Union nicht dennoch absegnet, lässt sich aus heutiger Sicht nicht beantworten. Gäbe der EuGH dem Druck nach, würde das im Falle deutscher Beteiligung sicherlich ein weiteres Verfahren vor dem Bundesverfassungsgericht nach sich ziehen, und zwar unter dem in dieser Konstellation ausnahmsweise durchaus Erfolg versprechenden Gesichtspunkt einer strukturwirksamen Verletzung der Kompetenzordnung.

\section{Fazit}

Die Eurokrise hat das politische und kulturelle Klima zwischen den Teilnehmerländern empfindlich gestört. Mit dem neuen makroökonomischen Verfahren wurde die Hoffnung auf frühzeitige Erkennung und Beseitigung wirtschaftlicher Ungleichgewichte verknüpft - eine Voraussetzung dafür, dass der Euro zu dem beitragen kann, wofür die europäische Integration doch letztlich antritt: die europäischen Länder und Völker einander näherzubringen. Allerdings führte unsere politökonomische und juristische Betrachtung der EIP zu ernüchternden Ergebnissen. Statt Harmonie zu stiften, dürfte die EIP neue Zwietracht säen, und mit ihr überschreitet die Union ihre Kompetenzen. Die wirtschaftlichen, politischen und in der Folge auch kulturellen Spannungen zwischen den Teilnehmerstaaten werden den Euro noch lange begleiten.

\section{Arne Heise}

\section{Governance auf verschiedenen Ebenen}

In den letzten zwei Jahren - die den Übergang von der vom amerikanischen Immobiliensektor ausgehenden Weltfinanzkrise zur Eurokrise markieren - haben reichlich 15 EU-(Sonder-)Gipfeltreffen stattgefunden. Im Zentrum dieser hektischen Suche nach Lösungen für die Eurokrise stand die Weiterentwicklung der europäischen Governance-Strukturen im Bereich der Wirtschafts- und
Finanzpolitik. Einerseits und vordringlich ging es um die Schaffung von Notfallverfahren, die den potenziellen Staatsbankrott einzelner Eurozonenländer - und damit wohl auch das Auseinanderbrechen der Europäischen Währungsunion (EWU) - verhindern sollen. Derartige Governance-Strukturen waren bei der Schaffung der EWU schlicht nicht vorgesehen, weil niemand - insbe- 